\title{
INSECT INSPIRED HEXAPOD ROBOT FOR TERRAIN NAVIGATION
}

\author{
Kale Aparna ${ }^{1}$, Salunke Geeta ${ }^{2}$ \\ ${ }^{1}$ M.E.Student, E\&Tc, G.S.Moze College of Engineering, Maharashtra, India, kaleaparna5@gmail.com \\ ${ }^{2}$ Professor, E\&Tc, AISSMS Institute of Information Technology, Maharashtra, India, geetasalunke@gmail.com
}

\begin{abstract}
The aim of this paper is to build a sixlegged walking robot that is capable of basicmobility tasks such as walking forward, backward, rotating in place and raising orlowering the body height. The legs will be of a modular design and will have threedegrees of freedom each. This robot will serve as a platform onto which additionalsensory components could be added, or which could be programmed to performincreasingly complex motions. This report discusses the components that make up ourfinal design.In this paper we have selected ahexapod robot; we are focusing \&developingmainly on efficient navigation method indifferent terrain using opposite gait of locomotion, which will make it faster and at sametime energy efficient to navigate and negotiate difficult terrain.This paper discuss the Features, development, and implementation of the Hexapod robot
\end{abstract}

Index Terms:Biologically inspired, Gait Generation,Legged hexapod, Navigation.

\section{INTRODUCTION}

In today's technological society, people have grown accustomed to daily use of several kinds of technology from personal computers to supercomputers, from personal vehicles to commercial airplanes, from mobile phones to communicating through the Internet and everything in between. As such, the use of robots has also become increasingly common. Robots can be used to complete repeated tasks, increase manufacturing production, carry extra weight and many other common tasks that humans do.

The hexapod robot has, by definition, six legs and is inspired by insects such as ants and crickets. This gives it the ability to move flexibly across various terrain, does not require any balancing mechanisms to stand upright. The main purpose for building this robot is to study the motions and movements of an insect. Applications for such arobot include environment exploration, search and rescue, and as a computer numerical control machine.

As the long term goal was to navigate the hexapod in debris or rubble, a special walking gait was created using force sensors to navigate on rough terrain.

\section{LITERATURE SURVEY}

Robots inspired by insects and other animals have previously been designed with physical antennae and tactile sensors to navigate their environment, as in the work by Brooks (1989)[10,12], Cowan et al. (2005), Hartmann (2001)[17] and Lee et al. (2008)[11]; the last three works employed the use of a single tactile element rather than a pair[3].
Because of their extreme mobility and agile adaptability to irregular terrain, insects have long been an inspiration for the designers of mobile and legged robots[1][4]. Early hexapod robots such as Genghis (Brooks, 1989)[20] and later creations such as Tarry (Frik et al., 1999)[20] implemented insect-like mobility based on observations of insect behaviors. The interleg coordination system developed by Holk Cruse (Cruse et al., 1991, 1998)[20,13] has been widely implemented in legged hexapods and its basis is in behavioral experiments that qualitatively analyzed insect walking behaviors[8].

Neurobiological control methods have previously been demonstrated for simulated hexapods (Ekeberg et al., 2004[16]; Lewinger et al., 2006[15]) and mechanical subsystems, such as single- and two-legged test platforms (Lewinger and Quinn, 2008)[14], but not yet as a complete and mechanical hexapod[2]. The hexapod described in this paperis the first physical and autonomous hexapod that uses a distributed walking control system based on the neurobiology of insects, stepping in the sagittal plane to angled stepping, which then induces turning in the robot.

\section{MODELLING}

Before any modeling, the coordinate systems for all parts of the robot need to be identified. All coordinate systems will be Cartesian and called frames[18].

\subsection{Robot body frame}

The origin of the robot coordinate frame will be in the center of the body, structured with Z-axis pointing up, the $\mathrm{X}$-axis positioning left and $\mathrm{Y}$-axis pointing forwards. 


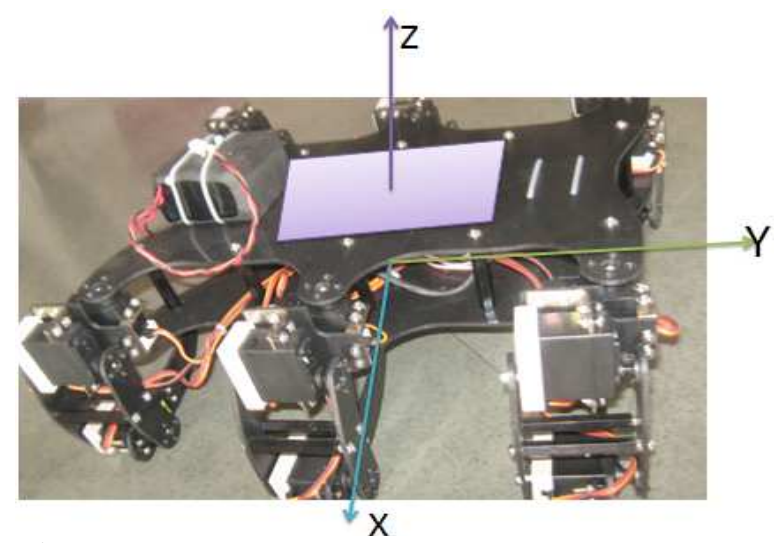

Fig -1: Location of body frame relative to robot hardware

\subsection{Leg frame and notations}

The coordinate frames for the robot legs are assigned as shown in fig1.The assignment of link frames follows the DenavitHartenberg notation. The robot leg is made of links and joints as noted on fig3, different links of robot leg are called Femur, Tibia and Tarsus. Fig 2 shows the 2D render of leg. The robot leg frame starts with link 0 which is the point where the leg is attached to the body, link 1 is Femur, link 2 is the Tibia and link 3 is Tarsus. The joints are located at the inner end of their respective link frames are attached to outer end of their respective links, this means that joint 2 rotates about the $\mathrm{Z}$-axis of frame 1 . The $\mathrm{Y}$-axis of links are not shown in Fig. 3 because they are irrelevant here.

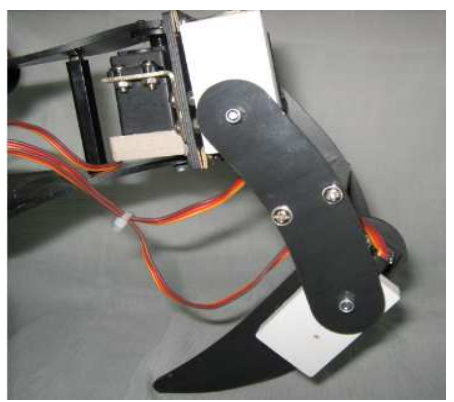

Fig- 2: 3D render for one leg

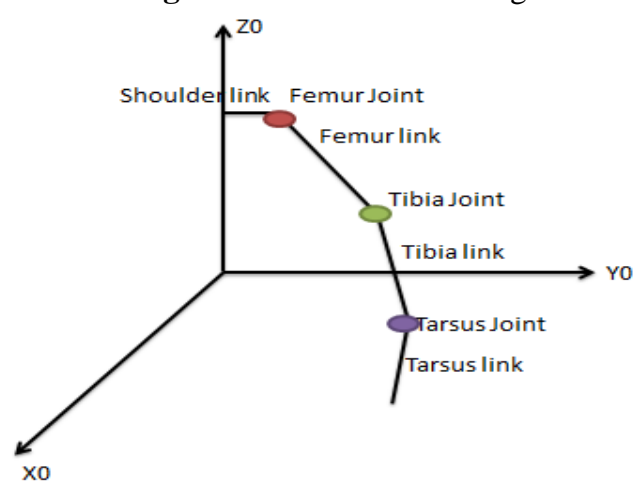

Fig-3: Isometric link view for leg links and joint

\subsection{Robot Leg Parameters}

The kinematic model here is derived by defining the reference frames according tothe Denavit-Hartenberg convention. In Fig.4 a graphical representation of a three joint robot legis given, with the attached reference frames and corresponding joint variables. In this figure the $\operatorname{body}(b)$ and the zeroth $(0)$ reference frames are attached to the stationary robot body. Therefore they canbe both considered as inertial frames. The axes of the body frame are arranged to be in accord with the actualrobot-body orientation. The Denavit-Hartenberg link parameters based on Fig.4 are given inTable 1.

The resulting homogeneous transformation matrices between the body and the zerothframe and between the sequential link frames are given in (1). In the formulas the variablesrepresented by aistand for the length of the ithlink (namely, the length of the portion of the linkbetween the origins of $i-1 s t$ and ithreference frames). The variables represented by $\theta i j$ mean the sumof the ithand jthjoint angles $(\theta i j=\theta i+\theta j) . \quad C$ and $S$ correspond to cosine and sinus functions,respectively. The values used for these parameters belong to the Hexapod robot: $\Psi=450 ; a 1=1.5(\mathrm{~cm}) ; a 2=5.25$ $(\mathrm{cm}) ; a 3=13.25(\mathrm{~cm})$.

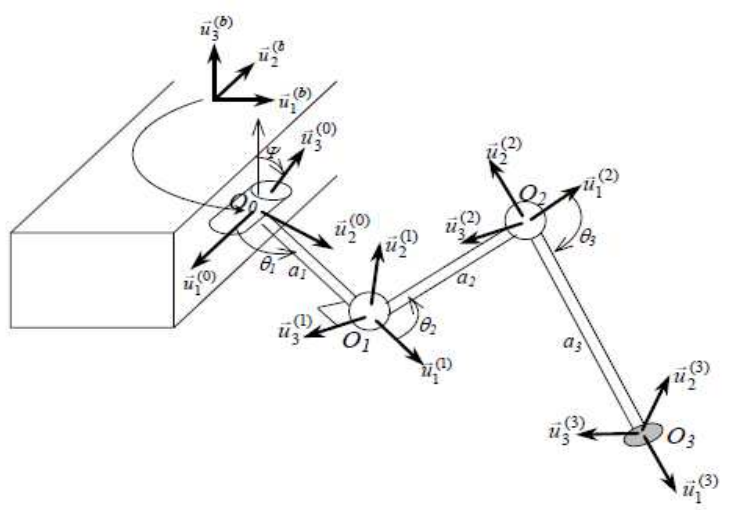

Fig- 4:Three-joint robot leg: Reference frames and joint variables.

Homogeneous matrices are used in derivation of positional relationsbetween the successive frames. In (3) the tip point position with respect to the body frame is given.The rotation matrices between the frames are given in (2). These rotation matrices are used invector equations, especially while deriving the dynamic equations.

Table -1: Denavit-Hartenberg link parameters for the three joint robot leg.

\begin{tabular}{|l|l|l|l|l|}
\hline Joint & $\boldsymbol{\theta} \mathrm{i}$ & $\alpha \mathrm{i}$ & $\mathrm{ai}$ & $\mathrm{di}$ \\
\hline 1 & $\boldsymbol{\theta} 1$ & $\pi / 2$ & $\mathrm{a} 1=1.5 \mathrm{~cm}$ & 0 \\
\hline 2 & $\boldsymbol{\theta} 2$ & 0 & $\mathrm{a} 2=5.25 \mathrm{~cm}$ & 0 \\
\hline 3 & $\boldsymbol{\theta} 3$ & 0 & $\mathrm{a} 3=13.25 \mathrm{~cm}$ & 0 \\
\hline
\end{tabular}




$$
\begin{aligned}
& \hat{H}^{(b, 0)}= {\left[\begin{array}{cccc}
0 & \cos (\Psi) & \sin (\Psi) & 0 \\
-1 & 0 & 0 & 0 \\
0 & -\sin (\Psi) & \cos (\Psi) & 0 \\
0 & 0 & 0 & 1
\end{array}\right] } \\
& \hat{H}^{(k-1, k)}= {\left[\begin{array}{cccc}
\cos \left(\theta_{k}\right) & -\sin \left(\theta_{k}\right) \cos \left(\alpha_{k}\right) & \sin \left(\theta_{k}\right) \sin \left(\alpha_{k}\right) & a_{k} \cos \left(\theta_{k}\right) \\
\sin \left(\theta_{k}\right) & \cos \left(\theta_{k}\right) \cos \left(\alpha_{k}\right) & -\cos \left(\theta_{k}\right) \sin \left(\alpha_{k}\right) & a_{k} \sin \left(\theta_{k}\right) \\
0 & \sin \left(\alpha_{k}\right) & \cos \left(\alpha_{k}\right) & d_{k} \\
0 & 0 & 0 & 1
\end{array}\right] } \\
& \widehat{H}^{(0,3)}=\widehat{H}^{(0,1)} \widehat{H}^{(1,2)} \widehat{H}^{(2,3)} \widehat{H}^{(b, 3)}=\widehat{H}^{(b, 0)} \widehat{H}^{(0,3)} \\
& \hat{C}^{(b, 0)}=\left[\begin{array}{cc}
0 & \cos (\psi) \sin (\psi) \\
-1 & 0 \\
0 & -\sin (\psi) \cos (\psi)
\end{array}\right] \\
& \hat{C}^{(k-1, k)}= {\left[\begin{array}{cc}
\cos \left(\theta_{k}\right)-\sin \left(\theta_{k}\right) \cos \left(\alpha_{k}\right)+\sin \left(\theta_{k}\right) \sin \left(\alpha_{k}\right) \\
\sin \left(\theta_{k}\right) \cos \left(\theta_{k}\right) \cos \left(\alpha_{k}\right)-\cos \left(\theta_{k}\right) \sin \left(\alpha_{k}\right) \\
0 & -\sin \left(\alpha_{k}\right) \cos \left(\alpha_{k}\right)
\end{array}\right] }
\end{aligned}
$$

$\overline{P_{e}^{(b)}} \overline{(\theta)}=\left[\begin{array}{c}C \psi\left(a_{1} S \theta_{1}+a_{2} S \theta_{1} C \theta_{2}+a_{3} S \theta_{1} C \theta_{23}\right)+S \psi\left(a_{2} S \theta_{2}+a_{3} S \theta_{23}\right) \\ -\left(a_{1} C \theta_{1}+a_{2} C \theta_{1} C \theta_{2}+a_{3} C \theta_{1} C \theta_{23}\right) \\ -S \psi\left(a_{1} S \theta_{1}+a_{2} S \theta_{1} C \theta_{2}+a_{3} S \theta_{1} C \theta_{23}\right)+C \psi\left(a_{2} S \theta_{2}+a_{3} S \theta_{23}\right)\end{array}\right]----$

In order to derive the dynamic equations, first the inertia matrices of the links should bedetermined. Since the $k$ threference frame is stationary with respect to the kthlink, the inertia tensorof the $k$ thlink around its center of mass appears to be a constant matrix with respect to the kthreference frame, as in (4). The values used in these formulations belong to the Hexapod robot. The resulting matricesfor each link are in the form of (5).

$\left\{\check{J}_{k}\right\}^{(k)}=\check{\jmath}_{k}^{(k)}=\check{J}_{k}$

$J_{k}=\left[\begin{array}{ccc}J_{k 1} & 0 & 0 \\ 0 & J_{k 2} & 0 \\ 0 & 0 & J_{k 3}\end{array}\right]$

The dynamic equations are derived by using the Lagrangian formulation. The Lagrange function $(L)$ is defined as the difference between the kinetic $(K)$ and potential $(U)$ energies. In the generalsense, the generalized forces of a conservative system are determined as in (6).

$L=K-U \quad \mathrm{~T}_{k}=\frac{d}{d t}\left(\frac{\partial L}{\partial q_{k}}\right)-\frac{\partial L}{\partial q_{k}}$

\section{STABILITY ANALYSIS OF STANDARD GAITS}

Considering the six legged walking, this paper proves that the wave gait is optimally stable among all periodic and regular gaits (the definitions are to be given) for all possible values of duty factor $(\beta)$, namely in the range of $1 / 2 \leq \beta<1$. The ninepages of proof for the single basic theorem in this paper clearly demonstrates how tedious it is to prove the optimal stability of wave gaits in the universe of periodic and regular gaits: the authors had to analyze 19 cases for derivation of the violating conditions, and then 31 cases to check these conditions for the six-legged case, and 5 cases to check them for the eight legged case; totally 55 cases are analyzed one by one[5][6].

Though the proof is very important to show the optimum stability of wave gaits in the determined ranges, it is overwhelmed by analysis of various particular cases, rather than narrowing down from the general to the optimum. The approach of the paper makes it difficult to get a comprehensive understanding of the nature of stable walk in multi-legged systems. In fact, it is possible to come up with a simpler and more insightful proof of the optimal stability of wave gaits for six (and four)-legged walking if the universe of search is limited to periodic, regular, and constant phase increment gaits, which are defined to be "standard gaits" .

\section{INVERSE KINEMATICS}

The forward kinematics is a simple equation used to calculate the position of the end effectors for the leg in the robot frame, by inserting the values of the angles for every joint. But the reverse operation is more complex, and it's is used to find all the angles of the joints only by knowing the position of the end effectors.

In general solving the IK equation can be a bit of a challenge. Some positions cannot be reached at all, as the physical system is unable to get there, and some end effectors positions can have more than one solution, and not all of them are desirable.

It is decided to solve the IK problem for each leg separately, as this makes it possible to solve it geometrically, by setting up some constrains .The first constraint for solving the IK equations and is given by the fact that all of the robots joints only allow rotation about one axis. The second constraint is that the Femur, Tibia and the Tarsus joints always rotate on parallel axes. The third set of constraints arises from the physical limitations for each joint, giving us some angular interval for each joint in which the servos can actually rotate the link. In Fig. 5 the limited angles of movement are shown.

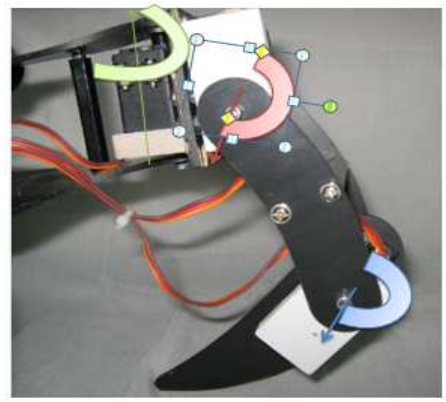

Fig-5: Illustration of the possible angles, which the legsjoints are confined to rotate within. 
First the coxa angle can be found easily using Atan2(X,Y) function, the relation between coxa angle and body is shown in Fig.6 this shows how coxa angle can be found directly by knowing the end effectors position.

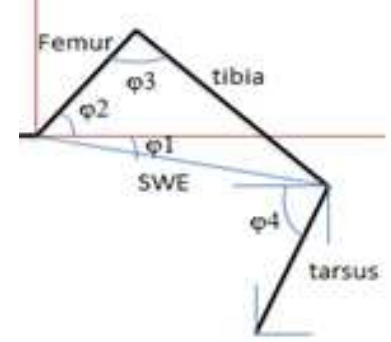

Fig-6: Illustration of the 2D triangle with vertices in the coxa, the femur, tibia and Tarsus link from origin

The equations below provide a summary of the formulas needed to find the individual joint angles.

$$
\begin{aligned}
& \theta 1=\operatorname{atan} 2(\mathrm{Y} 4, \mathrm{X} 4) \\
& \theta 2=\operatorname{acos} \frac{\left(\text { Femur }^{2}+S W E^{2}-\text { Tibia }^{2}\right)}{2 . F e m u r . S W E}+\operatorname{atan} 2(\mathrm{Y} 3, \mathrm{X} 3) \\
& \theta 3=180-\operatorname{acos} \frac{\left(\text { Femur }^{2}-S W E^{2}+\text { Tibia }^{2}\right)}{2 . \text { Femur. Tibia }} \\
& \boldsymbol{\theta} 4=\text { direct input angle that represent rotation to ground }
\end{aligned}
$$

\section{GAIT GENERATION}

In this section, the generation of the robot gait will be described. The robot generally has a group of programmed gait sequencesused for different purposes. For example a Tripedal gait is used as the basic movement for the robot which provide speed and longer traverse length, and a Metachronical gait is used for rough terrain traverse which provide better stability but slower motion[19,21]. Main types of gaits will be used in the robot:

\subsection{Wave Gait "Metachronical Gait"}

In this gait mode the robot move one leg at a time, it starts by lifting one leg and then lowering it down gradually until the foot touches the ground and then the next leg starts to move, as mentioned before this gait sequence is rather slow but it provides maximum stability for the robot, and it enables the robot to walk on rough terrain. This is illustrated in Fig.7.

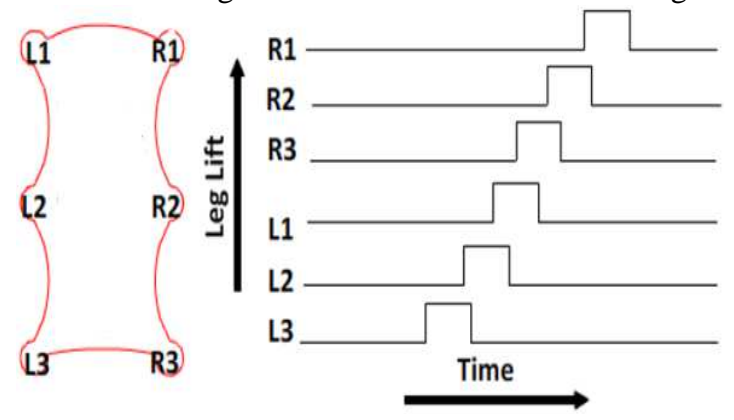

Fig-7: Single wave gait

\subsection{Ripple Gait "Two Wave Gait"}

In this gait, the two legs at a time, since it has two independent wave gaits. The opposite sides legs are 180 degrees out of phase and it needs three beats to complete one cycle. Fig. 8 shows the Ripple gait
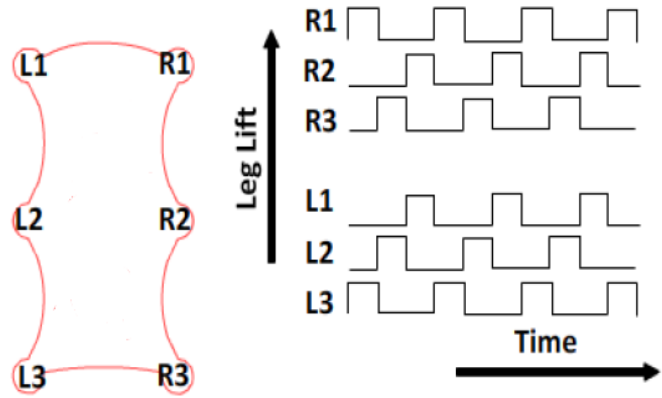

\subsection{Tripedal Gait}

Fig-8:Two wave gait

This gait is the fast gait for the hexapod; it completes a cycle in two beats. In this gait the robot lift three legs simultaneously while leaving three legs on the ground, which keeps the robot stable. Fig.9 shows Tripedal gait reaction[9].

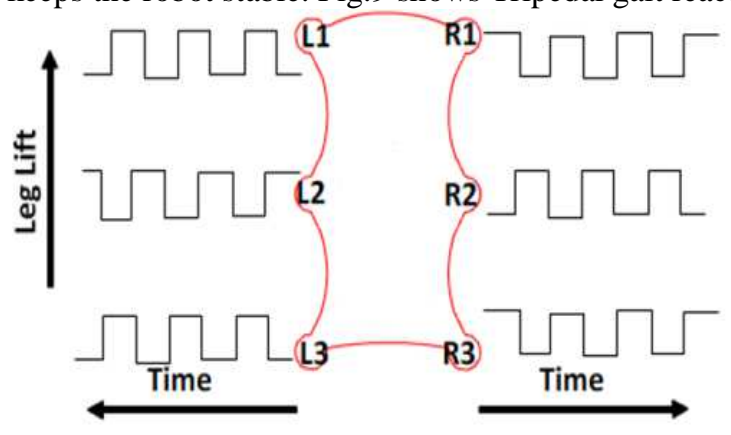

Fig-9: Tripedal gait

\section{JOINT ACTUATORS}

The leg joints are actuated by modified Quarter size servo Motor with Dual Ball Bearing and nylon Gears the Hitec HS805BB. These motors were chosen for their high torque.Because of their standard servo dimensions, these motors can be used with the wooden brackets, which minimized the number of custom-designed parts that were required. Each of the three joint actuators per leg directly drives its associated leg segment. By attaching the leg segment directly to the servo output horns, the mechanical design of the joints is simplified. This direct connection also allows the joints to take advantage of the nominal $\pm 90 \mathrm{deg}$ rangeofmotion (RoM) of the servos, which is approximately the same RoM as the insect[7].

It is important for the leg control system to know current joint angles (servo position) and joint loads (current consumption). As this information is not available from standard servos, the 
motors were modified. The servo's internal PCB, which is responsible for receiving PWM position commands from a host and converting those commands into servo output positions, has been removed, leaving the motor, transmission, and output potentiometer in the servo case. The two motor leads and three potentiometer leads are connected to a channel on the Motor Driver Board and Controller board.

\section{SYSTEM DESIGN AND DESCRIPTION}

However, the robot's static stability margin is not optimumwhen using gaits, for instance, five-leg support patternspresent better stability. Nevertheless, a hexapod configurationusing alternating tripods has been chosen just to try to increasethe machine's speed, albeit at the cost of slightly jeopardizingstability. To navigate in the hazardous field, it will rotate in any direction; heavy legs with powerful servomotors are chosen over other types as they can withstand heavy loads. It contains the required subsystems, such as, an ATMega Microcontroller, batteries, and play station receiver.

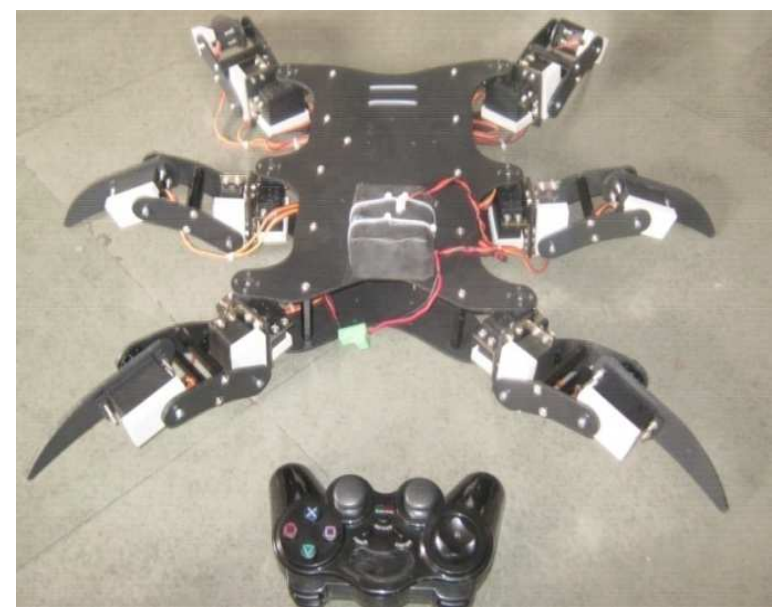

Fig-10:Hexapod configuration.

\subsection{Controller Board}

The Robot control board is versatile and expandable platform for robotics. The board is compatible to 6-25VDC input compared to all robot control boards available which accepts inputs just up to 12VDC.The motor drivers have inbuilt protection for overheating so it would not see motor drivers burnt in any case. The high capacity motor drivers give an edge to the development board for driving big robots without any problems.

The Quick C utilizes the in-circuit debugging capability built into theFlash devices. The In-Circuit Serial Programming (ICSP) protocol, offers costeffective, in-circuit flash debugging from the graphical user interface of the Quick $\mathrm{C}$ Integrated Development Environment. This enables a designer to develop and debug source code by setting breakpoints, single step- ping and watching variables, a CPU status and peripheral registers. Running at full speed enables testing hardware and applications in real time.

The play station transmitter sends commands to the robot, the play station receiver receives the commands and sends them to robot control board. The microcontroller contains all the necessarily algorithms such as Direct Servo Control, Forward Kinematics, Inverse Kinematics. After processing the input signal, it sends them to the servo driving circuit using TX/RX serial communication protocol.

The hexapod robot has custom design chassis, with six identical legs distributed evenly on the body. Each leg contains three-servo motor. And a total of 18 servo motors are used. The two servomotors are used vertically for joints 2 and joint 1 is configured around a typical rotary joint with horizontally constructed servo motor. With each leg configuration, we have all three-servo motors confined within a small volume near the body. The microcontroller reads the information and controls the movements of the robot.

\section{PHYSICAL ASPECTS}

The design of hexapod constitutes the kinematic configuration of a hexapodrobot, with each leg acting as an independent serialmanipulator with three degrees of freedom. Fig.11 showsthe actual prototype of our robot. The legs are numberedfrom 1 to 6 . Legs 1,2 , and 3 correspond to the legs on theright side of the robot's body while the left side legs arenumbered as 4,5 and 6 .

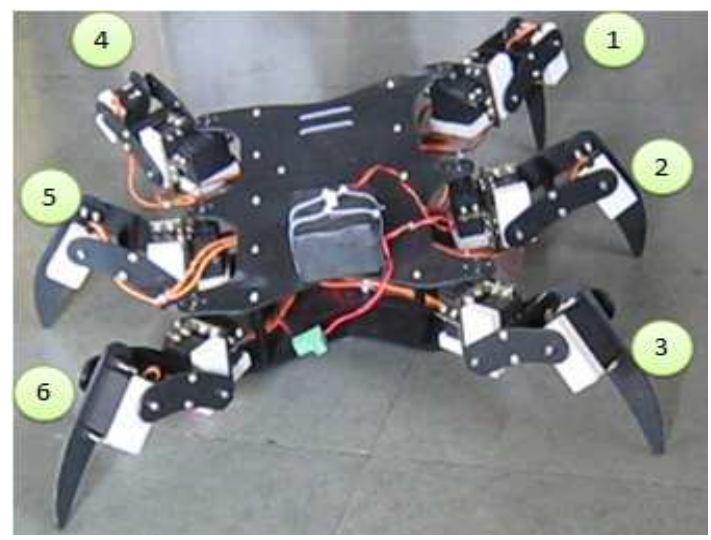

Fig- 11: Hexapod robot

Table -2: Physical characteristics of Hexapod 


\section{EXPERIMENTAL RESULTS}

A body of hexapod moves independently of its ground contact points. To makes a center of gravity shift on a horizontal plane, forward/backward, and sideways moving functions are effective. These functions can also produce a smooth body movement independently from an intermittent leg traveling.The robot has been designed with three degrees of freedom in the front, middle and rear legs respectively.From below figures, it is proved that the system has navigated the robot to walk according to the planned trajectory.

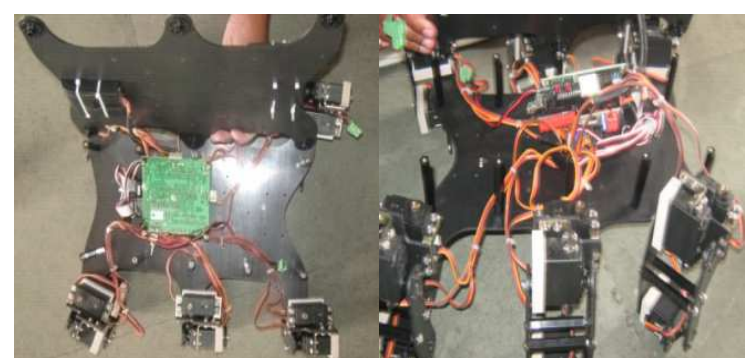

Fig- 12: Internal assembly of hexapod
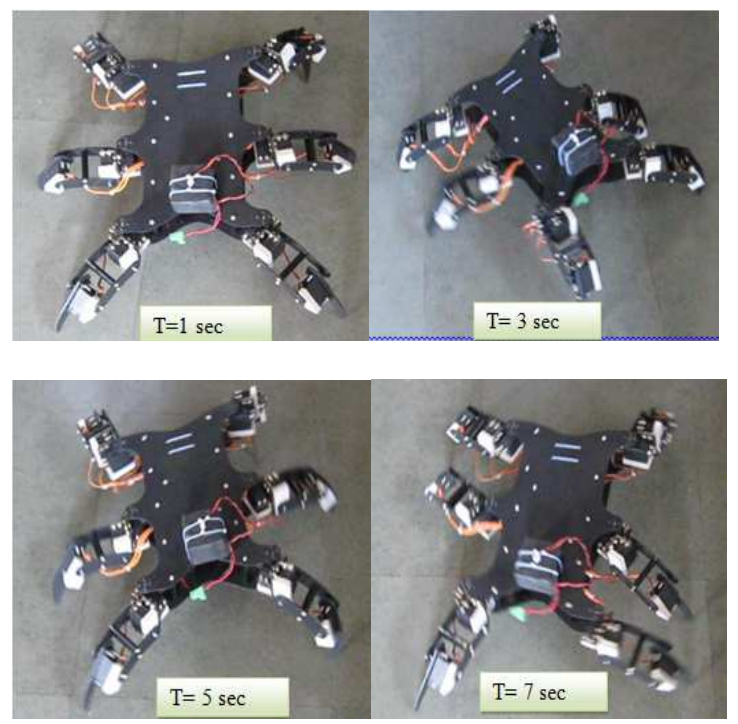

Fig- 13 : walking of hexapod

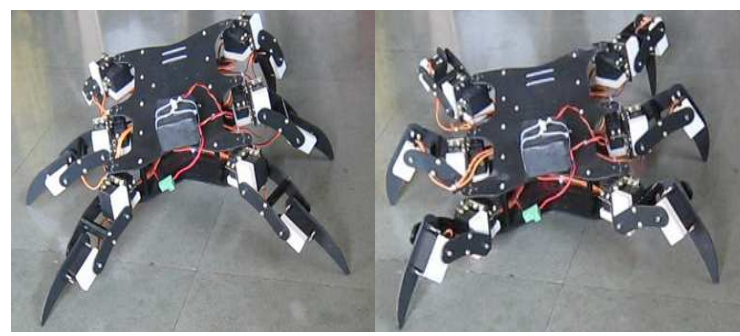

(a)

(b)

Fig-14.(a)Body rising movement(b) Body lowering movement

\begin{tabular}{|l|l|l|}
\hline Sr. & Parameter & Description \\
\hline 1 & Length & $30 \mathrm{~cm}$ \\
\hline 2 & Width & $23 \mathrm{~cm}$ \\
\hline 3 & Height & $12 \mathrm{~cm}$ \\
\hline 4 & Weight & $3 \mathrm{Kg}$ \\
\hline 5 & Construction Material & Fiber \\
\hline 6 & Actuators & DC servo motor \\
\hline 7 & Motion Control & ServoSequential Control \\
\hline 8 & Leg Stroke (Max) & $6 \mathrm{~cm}$ \\
\hline 9 & Leg Lift (Max) & $5 \mathrm{~cm}$ \\
\hline
\end{tabular}

\section{CONCLUSIONS}

The work presented here describes a hexapod robot based on the physical and neurobiological aspects of the Chrysolinafastuosabeetles. It has been designed to implement leg control methods as identified in the insect neurobiology and gait generation methods based on insect observations.Control of the leg actuators and execution of the leg control and gait generation models, is performed by a controller boards. The programmed speed and flexibility of these controllers make them ideal for this project and future research.

This paper describes the design \& simulation of the hexapod robot. The PWM joint controller has been shown to be capable of moving the joints with the necessary smoothness and precision. The results in simulation and on a real experimental hexapod robot show the feasibility and significance of the proposed controller.Here we have developed a method to perform terrain negotiation in a dynamic uncertain environment by using different gait locomotion. The algorithm we have developed for hexapod robots that may overcome many of the shortcomings of previous legged robots developed for hazardous field.

\section{ACKNOWLEDGEMENT}

The authors acknowledge the valuable technicalcontributions of Mr. Burse J.C. and the members ofthe Mechanical and Electronics Departments at theG. S. Moze College of Engineering, Pune, India.

\section{REFERENCES}

[1] W.A. Lewinger\& R.D. Quinn (2010) "A hexapod walks over irregular terrain using a controller adapted from an insects nervous system,"in proceeding of the IEEE/RSJ international conference on intelligent robots \& systems(IROS), Taiwan October 18-22,2010.

[2] FilippSeljanko(2011)"Hexapod walking Robot gait generation using genetic gravitational hybrid algorithm" in the $15^{\text {th }}$ International Conference on Advanced Robotics, Estonia,June 20-23,2011

[3] MohdDaud\&KenzoNonami(2012)"Autonomous walking over obstacles by means of LRF for hexapod robot 
COMET-IV"in journal of robotics \& Mechatronics Vol.24 no.1,2012

[4] William A.Lewinger, H.MartinReekie(2011)"A hexapod robot modeled on the stick insect,carausiusmorosus",in the $15^{\text {th }}$ international conference on advanced robotics,Tallinn,2011

[5] G.M.Nelson,R.D.Quinn(1997)"Design \& simulation of a crockroach like-Hexapod robot"in proceedings of the IEEE international conference on Robotics \& automation, New Mexico(1997)

[6] Hamid Mehdigholi\&SaeedAkbarnejad (2012)" Optimization of watt's six-bar linkage to generate straight \& parallel leg motion" in the journal of Humanoids,ISSN 1996-7209

[7]KanYoneda(2007)"Light weight quadruped with nine actuators" in journal of robotics \& mechatronics Vol.19 No.2,2007

[8] Dürr, V., Schmitz, J., Cruse, H. (2004), "Behaviour-based modeling of hexapod locomotion:linking biology and technical application". Arthropod Structure \& Development, 33: 237-250.

[9] Erden, M.S., Leblebicioğlu, K. (2005), "Multi legged walking in robotics and dynamic gaitpattern generation for a six-legged robot with reinforcement learning." book chapter in MobileRobots: New Research, Nova Publishers (in press, ISBN: 1-59454-359-3).

[10]Pashenkov, N. \&Iwamasa, R. / One-Chip Solution to Intelligent Robot Control: Implementing Hexapod Subsumption Architecture Using a Contemporary Microprocessor , pp. 93 - 98, International Journal of Advanced Robotic Systems, Volume 1 Number 2 (2004), ISSN 1729-8806

[11]Lee J, Sponberg S, LohO, CowanN. 2008. "Templates and anchorsfor antenna-based wall following in cockroaches and robots."IEEE Trans Robot. 24(6):130-143.

[12] Brooks, R. (1989), "A robot that walks: emergent behaviorsfrom a carefully evolved network", MIT AI Lab Memo1091, February.

[13]Cruse, H., Dean, J., Mu“ ller, U. and Schmitz, J. (1991), "The stick insect as a walking robot", Proceedings of the FifthInternational Conference on Advanced Robotics (ICAR'91),Pisa, Italy, Vol. 2, pp. 936-40.

[14]Lewinger, W.A. and Quinn, R.D. (2008), "BILL-LEGS: lowcomputation emergent gait system for small mobile robots",Proceedings of IEEE International Conference on Robotics andAutomation (ICRA'08), Pasadena, CA, May 11-23,pp. 251-6.

[15] W. A. Lewinger, "Insect-Inspired, Actively Compliant Robotic Hexapod," Department of Electrical Engineering and Computer Science, M.S. thesis, Case Western Reserve University, Cleveland, OH, 2005
[16]Ekeberg, O., Blümel, M., and Büschges, A. 2004.Dynamic simulation of insect walking.Arthropod Structure and Development 33:287-300 (Special Issue: Arthropod Locomotion Systems: from Biological Materials and Systems to Robotics, RE Ritzmann, SN Gorb and RD Quinn, eds.)

[17] Hartmann, M. J. (2001) "Active sensing capabilities of the rat whisker system", Autonomous Robots, 11:249-254

[18] "Introduction to Robotics: Mechanics \& control"J.Carig,John

[19] "Introduction to robotics"- SaeedB.Niku

[20]William A. Lewinger and Roger D. Quinn ,Neurobiologically-based control systemfor an adaptively walking hexapod doctoral diss., Case Western Reserve University, Cleveland, Ohio, USA, May 2012

[21]Hexapod robot gait, oricom technologies, www.oricomtech.com

\section{BIOGRAPHIES}

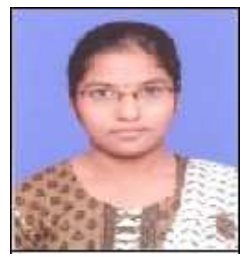

Ms. Kale Aparna received her B.E. degree in Electronics engineering from Shivaji University of Maharashtra, India. She is the post graduate research assistant at G.S.Moze College of engineering; her current research topic is Multilegged Locomotion on Terrain surface.

Prof. Salunke Geeta is currently working as Professor in Electronics

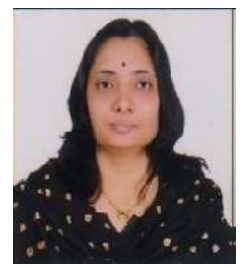
and Telecommunication at AISSMS Institute of information technology, Pune. She has vast teaching experience and published numbers of papers in international, national Journals and conferences. Her area of research is image processing, robotics and neural network. 